International Mathematical Forum, Vol. 9, 2014, no. 22, 1061 - 1073

HIKARI Ltd, www.m-hikari.com

http://dx.doi.org/10.12988/imf.2014.45102

\title{
New Expansion and Infinite Series
}

\author{
Daiyuan Zhang \\ College of Computer \\ Nanjing University of Posts and Telecommunications \\ Nanjing, P.R. China
}

Copyright (c) 2014 Daiyuan Zhang. This is an open access article distributed under the Creative Commons Attribution License, which permits unrestricted use, distribution, and reproduction in any medium, provided the original work is properly cited.

\begin{abstract}
Different from the Taylor polynomials, a new formula for function expansion is proposed where the terms are not polynomials. A new infinite series based on the new formula is also proposed, and the new infinite series can keep some important properties of the original functions. Some forms of remainder are also presented for analysis of convergence. In order to show some internal relationships between the new results and Taylor's theorem, some important theorems have been proved in this paper. Finally, Some examples are given and the regions of convergence for the new infinite series are analyzed, the results show that the region of convergence is much larger than that obtained by Taylor's Series.
\end{abstract}

Mathematics Subject Classification: 41A58, 41A20, 41A30, 40A30

Keywords: Expansion of function, Taylor expansion, Taylor series, Remainder analysis, Convergence, Region of convergence

\section{Introduction}

It is well known that Taylor's theorem $[1,2]$ has many applications in mathematics, physics, engineering and other fields. Up to now, many researches are also related with Taylor's expansion or Taylor's series. For example, the approximate method for solving nonlinear differential equations [3]. In [4], several examples were presented for ordinary differential equation applications 
that can be solved successfully using the Taylor series method. In [5], A numerical method for solving partial differential equations based upon the Taylor series was proposed. Literature [6] presented a Taylor-series expansion method for a second kind Fredholm integral equations system. In [7], by using a special property in the integral representation of the remainder value of the Taylor series expansion, an expansion for analytic functions was introduced. In literature [8], the research explicitly obtain the relation between the coefficients of the Taylor series and Jacobi polynomial expansions.

Although some applications have been mentioned above, there are many other applications about Taylor's expansion or series.

Let $f(x)$ be a given function, The results mentioned above are all related with original form of Taylor's expansion or series, in which the coefficients of each term are always the constant derivatives $f^{(k)}(a)$ at a point $a$, and no matter how many terms of Taylor's expansion you choose, they are a kind of polynomials of $x$ when neglecting the remainder.

Although the Taylor theorem is important and has many applications, we have to ask a question: are there any other forms of expansion for a given function $f(x)$ besides the original form of Taylor's expansion? The answer is "yes".

In this paper, a new form of expansion is proposed, which is different from the original form of Taylor's expansion. In general, the new expansion generated by a given function $f(x)$ is not the form of the Taylor polynomials of the variable $x$. For the given function $f(x)$, the terms generated by the new expansion are of functions associated with the derivatives $f^{(k)}(x)$ of the first $k$ orders, instead of the constant derivatives $f^{(k)}(a)$ at a point $a$. In some cases, the expansions generated by the theorem proposed in this paper are rational functions, instead of polynomial functions. In many applications, we have to deal with the functions with poles, such as rational functions, logarithmic functions and so on, but the polynomial functions can not express the properties of poles and corresponding singular parts properly.

A new infinite series based on the expansion is also proposed in this paper, and the region of convergence of the infinite series is analyzed. Some application examples showed that the region of convergence is much larger than that obtained by the original form of Taylor's series directly. Although there are many ways to approximate a given function $f(x)$ by polynomials, rational functions or some other forms of functions, the method proposed in this paper is a special one, depending on the functions of derivatives.

The new expansion and infinite series may be widely used in the fields to which the original form of Taylor's expansion and infinite series can be applied. Using the new results, we may obtain more achievements in many fields. 


\section{New Formula of Expansion}

Let $f(x)$ be a function, the entire real line is denoted by $\mathbb{R}$. We define $C^{n}[A, B]$ to be the set of functions for which $f^{(n)}(x)$ exists and is continuous on the closed interval $[A, B] \subseteq \mathbb{R}$.

An important theorem concerning a new expansion for a function is proposed in this paper and given in the following:

Theorem 2.1. Let $f(x)$ be a function having finite $(n+1)$ th derivative $f^{(n+1)}(x)$ everywhere in an open interval $(A, B)$ and assume that $f(x) \in$ $C^{n}[A, B]$. Assume that $a \in[A, B]$. Then, for every $x$ in $[A, B]$, we have

$$
f(x)=Z_{n}[f(x ; a)]+R_{n}[f(x ; a)]
$$

where

$$
\begin{gathered}
Z_{n}[f(x ; a)]=f(a)+\sum_{k=1}^{n}(-1)^{k-1} \frac{f^{(k)}(x)}{k !}(x-a)^{k} \\
R_{n}[f(x ; a)]=\frac{1}{n !} \int_{a}^{x}(a-t)^{n} f^{(n+1)}(t) d t
\end{gathered}
$$

where $Z_{n}[f(x ; a)]$ is known as the expansion of function $f(x)$ up to the $n$-th derivative at the point $x=a$, and $R_{n}[f(x ; a)]$ is known as the remainder of the expansion $Z_{n}[f(x ; a)]$ at the point $x=a$.

Proof. Let us consider the following integral:

$$
I_{n}=\frac{1}{n !} \int_{a}^{x}(t-a)^{n} f^{(n+1)}(t) d t
$$

integrating by parts we find that

$$
\begin{aligned}
& I_{n}=\frac{1}{n !} \int_{a}^{x}(t-a)^{n} f^{(n+1)}(t) d t=\frac{1}{n !} \int_{a}^{x}(t-a)^{n} d f^{(n)}(t) \\
& =\frac{1}{n !}(x-a)^{n} f^{(n)}(x)-\frac{1}{(n-1) !} \int_{a}^{x}(t-a)^{n-1} f^{(n)}(t) d t
\end{aligned}
$$

From (5) we find the recursive formula in the following:

$$
I_{n}=\frac{1}{n !}(x-a)^{n} f^{(n)}(x)-I_{n-1}
$$

From (4) we have

$$
I_{0}=f(x)-f(a)
$$


From (6) we have

$$
I_{n}=\frac{1}{n !}(x-a)^{n} f^{(n)}(x)-\frac{1}{(n-1) !}(x-a)^{n-1} f^{(n-1)}(x)+I_{n-2}
$$

When $n$ is odd, using (8), we have

$$
I_{n}=\frac{f^{(n)}(x)}{n !}(x-a)^{n}-\frac{f^{(n-1)}(x)}{(n-1) !}(x-a)^{n-1}+\cdots+f^{\prime}(x)(x-a)-I_{0}
$$

Hence

$$
I_{0}=\sum_{k=1}^{n}(-1)^{k-1} \frac{f^{(k)}(x)}{k !}(x-a)^{k}-I_{n}
$$

Therefore, when $n$ is odd, from (4), (7) and (10) we have

$$
f(x)=f(a)+\sum_{k=1}^{n}(-1)^{k-1} \frac{f^{(k)}(x)}{k !}(x-a)^{k}+\frac{1}{n !} \int_{a}^{x}(a-t)^{n} f^{(n+1)}(t) d t
$$

When $n$ is even, from (8) and (6), we get

$$
I_{n}=\frac{f^{(n)}(x)}{n !}(x-a)^{n}-\frac{f^{(n-1)}(x)}{(n-1) !}(x-a)^{n-1}+\cdots-f^{\prime}(x)(x-a)+I_{0}
$$

then

$$
I_{0}=\sum_{k=1}^{n}(-1)^{k-1} \frac{f^{(k)}(x)}{k !}(x-a)^{k}+I_{n}
$$

Therefore, when $n$ is even, from (4), (7) and (13) we have

$$
f(x)=f(a)+\sum_{k=1}^{n}(-1)^{k-1} \frac{f^{(k)}(x)}{k !}(x-a)^{k}+\frac{1}{n !} \int_{a}^{x}(a-t)^{n} f^{(n+1)}(t) d t
$$

From (14) and (11), we can conclude that, no matter what the number of $n$ may be (odd or even), the following always holds:

$$
f(x)=f(a)+\sum_{k=1}^{n}(-1)^{k-1} \frac{f^{(k)}(x)}{k !}(x-a)^{k}+\frac{1}{n !} \int_{a}^{x}(a-t)^{n} f^{(n+1)}(t) d t
$$

This completes the proof.

If ignoring the remainder of (15), we have the following approximate formula

$$
f(x) \approx Z_{n}[f(x ; a)]=f(a)+\sum_{k=1}^{n}(-1)^{k-1} \frac{f^{(k)}(x)}{k !}(x-a)^{k}
$$


Formula (2) (or(16)) is different from Taylor's theorem. The right-hand side of formula (16) is a new form of expansion generated by a given function $f(x)$ at a point $a$. Obviously, $f^{(k)}(x)$ is usually not a polynomial for a given function $f(x)$, the right-hand side of formula (16) is usually not a polynomial either, therefore, we obtain a new form of expansion generated by a given function $f(x)$ at a point $a$. Taylor's theorem gives an approximation of a $k$ times differentiable function around a given point by a $k$-th order Taylor polynomial, which approximately determines the function in some neighborhood of the point. But our formula (see(2) or (16)) shows that, in some applications, we give an approximation of a $k$ times differentiable function around a given point by a kind of function rather than polynomial, for example, some rational functions are obtained (see examples of section 6). Obviously, rational function is usually different from polynomial, therefore, our formula (2) (or(16)) is a new formula.

It should be noted that, in our formula (2) or (16), the coefficients $(-1)^{k-1} f^{(k)}(x) / k$ ! $(k=1,2, \cdots, n)$ of terms $(x-a)^{k}$ are functions of variable $x$, which is different from the constant coefficients $f^{(k)}(a) / k$ ! obtained by Taylor's expansion.

\section{Forms of Remainder}

\subsection{The First Form of Remainder}

When $x>a,(a-x)^{n}$ never changes sign on $[a, x]$ and assume $f^{(n+1)}(x)$ is continuous on $[a, x]$, then, using the generalized Mean-Value Theorem [1] in (3), we have

$$
R_{n}[f(x ; a)]=\frac{1}{n !} \int_{a}^{x}(a-t)^{n} f^{(n+1)}(t) d t=(-1)^{n} \frac{f^{(n+1)}(\xi)}{(n+1) !}(x-a)^{n+1}
$$

Or

$$
\left|R_{n}[f(x ; a)]\right|=\frac{\left|f^{(n+1)}(\xi)\right|}{(n+1) !}|x-a|^{n+1}
$$

where $\xi$ lies between $a$ and $x$.

When $x<a,(a-x)^{n}$ also never changes sign on $[x, a]$, we also have the same results like (17) and (18).

When $x=a$, the right-hand side of integral (3) is 0 , the right-hand side of (18) is also 0. Therefore, no matter what happens, (18) always holds. 


\subsection{The Second Form of Remainder}

Since $(a-t)^{n}$ is continuous function of $t$, assume $f^{(n+1)}(t)$ is continuous on $[a, x]$, using the Mean-Value Theorem in (3), we have

$$
R_{n}[f(x ; a)]=\frac{1}{n !} \int_{a}^{x}(a-t)^{n} f^{(n+1)}(t) d t=\frac{(a-\xi)^{n}}{n !} f^{(n+1)}(\xi)(x-a)
$$

where $\xi$ lies between $a$ and $x$.

\subsection{The Third Form of Remainder}

Since $(a-t)^{n}$ is continuous function of $t$, assume $f^{(n+1)}(t)$ is continuous and never changes sign on $[a, x]$, by using the generalized Mean-Value Theorem in (3), we have

$$
\begin{aligned}
R_{n}[f(x ; a)] & =\frac{1}{n !} \int_{a}^{x}(a-t)^{n} f^{(n+1)}(t) d t=\frac{(a-\xi)^{n}}{n !} \int_{a}^{x} f^{(n+1)}(t) d t \\
& =\frac{(a-\xi)^{n}}{n !}\left(f^{(n)}(x)-f^{(n)}(a)\right)
\end{aligned}
$$

where $\xi$ lies between $a$ and $x$.

\section{Infinite Series Based on the New Expansion}

Suppose that $f(x)$ is a function all of whose differential functions $f^{(k)}(x)$ are continuous in an interval surrounding the point $x=a$. Let $n \rightarrow \infty$ in formula (2), we can certainly form the infinite series in the following and denoted by $Z_{\infty}[f(x ; a)]:$

$$
Z_{\infty}[f(x ; a)]=f(a)+\sum_{k=1}^{\infty}(-1)^{k-1} \frac{f^{(k)}(x)}{k !}(x-a)^{k}
$$

The expression (21) is called the infinite series based on the new expansion (2), which is generated by $f(x)$ at $a$. If $a=0$, we have

$$
Z_{\infty}[f(x ; 0)]=f(0)+\sum_{k=1}^{\infty}(-1)^{k-1} \frac{f^{(k)}(x)}{k !} x^{k}
$$




\section{Convergence Analysis of the New Infinite Series}

We have proved that the formula of remainder (error term) could be expressed as integral (3), in which $f^{(n+1)}(x)$ is continuous. Therefore, we have the following theorem.

Theorem 5.1. If $f(x)$ is infinitely differentiable in a set $\mathbb{S}, x \in \mathbb{S}, a \in \mathbb{S}$, the series (21) converges to $f(x)$ if and only if integral (3) tends to 0 as $n \rightarrow \infty$, i.e.

$$
\lim _{n \rightarrow \infty} R_{n}[f(x ; a)]=0
$$

Integral (3) is called the integral form of the remainder. $\lim _{n \rightarrow \infty} R_{n}[f(x ; a)]$ is also denote by $R_{\infty}[f(x ; a)]$. The form of integral (3) enables us to give the following sufficient condition for convergence of the series proposed in this paper.

Theorem 5.2. Assume $f(x)$ is infinitely differentiable in a set $\mathbb{S}, x \in \mathbb{S}$, $a \in \mathbb{S}$, and assume that there is a positive constant $M$ such that

$$
f^{(n)}(x) \leqslant M, n=1,2, \cdots, x \in \mathbb{S}, a \in \mathbb{S}
$$

then the series generated by $f(x)$ at a (see(21)) converges to $f(x)$ for each $x \in \mathbb{S}, a \in \mathbb{S}$, i.e.

$$
f(x)=Z_{\infty}[f(x ; a)], x \in \mathbb{S}, a \in \mathbb{S}
$$

Proof. Using (18) and (24), we obtain the estimate

$$
\left|R_{n}[f(x ; a)]\right| \leqslant \frac{M}{(n+1) !}|x-a|^{n+1}
$$

But $|x-a|^{n+1} /(n+1) ! \rightarrow 0$ as $n \rightarrow \infty$ for each finite $x \in \mathbb{S}, a \in \mathbb{S}$, hence

$$
\left|R_{\infty}[f(x ; a)]\right|=\left|\lim _{n \rightarrow \infty} R_{n}[f(x ; a)]\right|=0
$$

i.e.

$$
R_{\infty}[f(x ; a)]=0, x \in \mathbb{S}, a \in \mathbb{S}
$$

therefore, $f(x)=Z_{\infty}[f(x ; a)]+R_{\infty}[f(x ; a)]=Z_{\infty}[f(x ; a)], x \in \mathbb{S}, a \in$ $\mathbb{S}$.

In order to show some internal relationships between the new results proposed in this paper and Taylor's theorem, we write Taylor's theorem in the following:

$$
f(x)=T_{n}[f(x ; a)]+E_{n}[f(x ; a)]
$$


where

$$
\begin{gathered}
T_{n}[f(x ; a)]=f(a)+\sum_{k=1}^{n} \frac{f^{(k)}(a)}{k !}(x-a)^{k} \\
E_{n}[f(x ; a)]=\frac{1}{n !} \int_{a}^{x}(x-t)^{n} f^{(n+1)}(t) d t
\end{gathered}
$$

We denote $\lim _{n \rightarrow \infty} T_{n}[f(x ; a)]$ and $\lim _{n \rightarrow \infty} E_{n}[f(x ; a)]$ by $T_{\infty}[f(x ; a)]$ and $E_{\infty}[f(x ; a)]$ respectively. Taylor's series can be written in the following:

$$
T_{\infty}[f(x ; a)]=f(a)+\sum_{k=1}^{\infty} \frac{f^{(k)}(a)}{k !}(x-a)^{k}
$$

Theorem 5.3. When $x \in \mathbb{S}, a \in \mathbb{S}$, if $R_{\infty}[f(x ; a)]=0$ and $E_{\infty}[f(x ; a)]=$ 0 , then the following holds

$$
f(x)=\lim _{n \rightarrow \infty} Z_{n}[f(x ; a)]=\lim _{n \rightarrow \infty} T_{n}[f(x ; a)]
$$

Or

$$
f(x)=Z_{\infty}[f(x ; a)]=T_{\infty}[f(x ; a)]
$$

Proof. Since $R_{\infty}[f(x ; a)]=0, x \in \mathbb{S}, a \in \mathbb{S}$, from Theorem 5.1 we have $f(x)=Z_{\infty}[f(x ; a)], x \in \mathbb{S}, a \in \mathbb{S}$. Let $n \rightarrow \infty$, from (29) we have

$$
f(x)=T_{\infty}[f(x ; a)]+E_{\infty}[f(x ; a)]=T_{\infty}[f(x ; a)]
$$

Therefore, expressions (33) and (34) hold.

Theorem 5.3 indicates that, if the conditions of Theorem 5.3 are satisfied, the series $Z_{\infty}[f(x ; a)]$ (proposed in this paper) and Taylor's series $T_{\infty}[f(x ; a)]$ are equal and they both converge to $f(x)$ for each $x \in \mathbb{S}, a \in \mathbb{S}$.

Generally speaking, if $x \notin \mathbb{S}$, the series $Z_{\infty}[f(x ; a)]$ (proposed in this paper) will have different values from Taylor's series $T_{\infty}[f(x ; a)]$, which indicate that the series (proposed in this paper) is a new kind of infinite series. More detail will be given in Example 6.1 and Example 6.2.

Theorem 5.4. Let $M$ be a positive constant, $0<M<\infty$, if $\left|f^{(n)}(x)\right|<M$ for any $x \in(-\infty, \infty), n=1,2, \cdots$, then the following holds:

$$
f(x)=Z_{\infty}[f(x ; a)]=T_{\infty}[f(x ; a)], a \in(-\infty, \infty), x \in(-\infty, \infty)
$$

Proof. Let $\mathbb{S}=(-\infty, \infty)$, from Theorem 5.2 we have $f(x)=Z_{\infty}[f(x ; a)], a \in$ $(-\infty, \infty), x \in(-\infty, \infty)$. On the other hand, let $\xi=a+\theta(x-a), 0<\theta<1$, 
then $\xi$ must lie between $x$ and $a$, using the Mean-Value Theorem in (31), we have

$$
\begin{aligned}
E_{n}[f(x ; a)] & =\frac{1}{n !} \int_{a}^{x}(x-t)^{n} f^{(n+1)}(t) d t=\frac{1}{n !}(x-\xi)^{n} f^{(n+1)}(\xi)(x-a) \\
& =\frac{(x-a)^{n+1}}{n !}(1-\theta)^{n} f^{(n+1)}(a+\theta(x-a))
\end{aligned}
$$

Since $0<\theta<1,\left|f^{(n+1)}(a+\theta(x-a))\right|<M$ and $(x-a)^{n+1} / n ! \rightarrow 0$ as $n \rightarrow \infty$, then we obtain $E_{\infty}[f(x ; a)]=0$. Let $n \rightarrow \infty$, from (29) we have

$f(x)=T_{\infty}[f(x ; a)]+E_{\infty}[f(x ; a)]=T_{\infty}[f(x ; a)], a \in(-\infty, \infty), x \in(-\infty, \infty)$

Therefore, (35) holds.

\section{Examples}

Some examples will be given in the following for describing the applications of the results proposed in this paper.

Example 6.1 Let $x \neq a, a>0, x>0, f(x)=\ln (x)$, using Theorem 2.1, we have:

$$
\ln (x)=\ln (a)+\sum_{k=1}^{n}(-1)^{k-1} \frac{\ln ^{(k)}(x)}{k !}(x-a)^{k}+R_{n}[f(x ; a)]
$$

where

$$
R_{n}[f(x ; a)]=\frac{1}{n !} \int_{a}^{x}(a-t)^{n} \ln ^{(n+1)}(t) d t
$$

Since

$$
\ln ^{(k)}(x)=(-1)^{k-1}(k-1) ! x^{-k}
$$

Then, formula (37) can be written in the following

$$
\ln (x)=\ln (a)+\sum_{k=1}^{n} \frac{1}{k}\left(\frac{x-a}{x}\right)^{k}+\int_{a}^{x}(t-a)^{n} t^{-(n+1)} d t
$$

The infinite series $Z_{\infty}[f(x ; a)]$ is

$$
Z_{\infty}[f(x ; a)]=Z_{\infty}[\ln (x ; a)]=\ln (a)+\sum_{k=1}^{\infty} \frac{1}{k}\left(\frac{x-a}{x}\right)^{k}
$$


Using (19), we have

$$
\begin{aligned}
R_{\infty}[f(x ; a)] & =\lim _{n \rightarrow \infty} R_{n}[f(x ; a)]=\lim _{n \rightarrow \infty} R_{n}[\ln (x ; a)] \\
& =\lim _{n \rightarrow \infty} \frac{1}{n !} \int_{a}^{x}(a-t)^{n} \ln ^{(n+1)}(t) d t \\
& =\lim _{n \rightarrow \infty}\left((-1)^{n} \frac{(a-\xi)^{n}}{\xi^{n+1}}(x-a)\right) \\
& =\lim _{n \rightarrow \infty}\left(\left(\frac{\theta(x-a)}{a+\theta(x-a)}\right)^{n} \frac{(x-a)}{(a+\theta(x-a))}\right)
\end{aligned}
$$

where $0<\theta<1$.

Since $a>0,0<\theta<1$, when $\theta(x-a)>-a / 2$, the value of (42) tends to 0 , or when

$$
x>a-\frac{a}{2 \theta}=\left(1-\frac{1}{2 \theta}\right) a
$$

is satisfied, we get $R_{\infty}[f(x ; a)]=\lim _{n \rightarrow \infty} R_{n}[f(x ; a)]=0$.

Since $a>0,0<\theta<1$, the range of $(1-1 /(2 \theta)) a$ (see the right-hand side of $(43))$ is $(-\infty, a / 2)$, if we choose

$$
x \geqslant a\left(1-\frac{1}{2}\right)=\frac{a}{2}
$$

then (43) must be satisfied.

Therefore, when $x \geqslant a / 2, a>0$, the function $\ln (x)$ can be expressed by the following convergent series.

$$
\ln (x)=Z_{\infty}[\ln (x ; a)]=\ln (a)+\sum_{k=1}^{\infty} \frac{1}{k}\left(\frac{x-a}{x}\right)^{k}, x \geqslant a / 2, a>0
$$

Let $a=1$, we have

$$
\ln (x)=\sum_{k=1}^{\infty} \frac{1}{k}\left(\frac{x-1}{x}\right)^{k}, x \in[1 / 2, \infty)
$$

The Taylor series for function $\ln (x)$ can be written in the following

$$
\ln (x)=(x-1)-\frac{(x-1)^{2}}{2}+\cdots=\sum_{k=1}^{\infty}(-1)^{k+1} \frac{(x-1)^{k}}{k}, x \in(0,2]
$$

The region of convergence of Taylor's series is $x \in(0,2]$, which is much less than the region $(x \in[1 / 2, \infty))$ obtained in this paper. 
If $x \in([1 / 2, \infty) \cap(0,2])$, or $x \in[1 / 2,2]$, the series (46) and Taylor's series (47) are equal and converge to $\ln (x)$ (see Theorem 5.3), but when $x \notin$ $([1 / 2, \infty) \cap(0,2])$ or $x \notin[1 / 2,2]$, the series (46) and Taylor's series (47) will have different values, therefore, they are different functions.

Generally speaking, the series $Z_{\infty}[f(x ; a)]$ (proposed in this paper) is different from Taylor's series $T_{\infty}[f(x ; a)]$, which indicate that the series $Z_{\infty}[f(x ; a)]$ (proposed in this paper) is a new kind of infinite series.

On the other hand, although the point $x=0$ is divergent both in the righthand side of series (46) and (47), series (46) shows an important property that the point $x=0$ is a pole of the series $Z_{\infty}[\ln (x ; a)]$ (see the right-hand side of (46)), which is also the pole of $\ln (x)$, while in series (47), the pole of $\ln (x)$ is not the pole of Taylor's series (see the right-hand side of (47)). It implies that Taylor's series may lose some important properties of the original functions.

Example 6.2 Let $f(x)=1 /(x+b), a \neq-b$, from (1), (2), (3) we have

$$
f(x)=Z_{n}[f(x ; a)]+R_{n}[f(x ; a)]
$$

where

$$
Z_{n}[f(x ; a)]=\frac{1}{a+b}-\sum_{k=1}^{n} \frac{(x-a)^{k}}{(x+b)^{k+1}}
$$

From (19) we have

$$
R_{n}[f(x ; a)]=-\left(\frac{\theta(x-a)}{a+\theta(x-a)+b}\right)^{n} \frac{(n+1)(x-a)}{(a+\theta(x-a)+b)^{2}}
$$

Then

$$
R_{\infty}[f(x ; a)]=-\lim _{n \rightarrow \infty}\left(\left(\frac{\theta(x-a)}{a+\theta(x-a)+b}\right)^{n} \frac{(n+1)(x-a)}{(a+\theta(x-a)+b)^{2}}\right)
$$

where $0<\theta<1$.

If $a+b>0$, when $\theta(x-a)>-1 /(2(a+b))$, from $(51)$, we have $\lim _{n \rightarrow \infty} R_{n}[f(x ; a)]=$ 0 , or when

$$
x>a-\frac{1}{2(a+b) \theta}
$$

is satisfied, the following series (53) is convergent.

$$
Z_{\infty}[f(x ; a)]=\lim _{n \rightarrow \infty} Z_{n}[f(x ; a)]=\frac{1}{a+b}-\sum_{k=1}^{\infty} \frac{(x-a)^{k}}{(x+b)^{k+1}}
$$

Since $0<\theta<1$, the range on the right-hand side of $(52)$ is $(-\infty, a-1 /(2(a+b)))$, if we choose

$$
x \geqslant a-\frac{1}{2(a+b)}
$$


then the inequality (52) always holds. Therefore, when $a+b>0$, the series (53) generated by function $f(x)=1 /(x+b)$ is convergent if (54) holds.

If $a+b<0$, when $\theta(x-a)<1 /(2(a+b))$, we have $\lim _{n \rightarrow \infty} R_{n}[f(x ; a)]=0$ from (51), or when

$$
x<a-\frac{1}{2(a+b) \theta}
$$

is satisfied, the series (53) is convergent.

Since $0<\theta<1$, the range on the right-hand side of $(55)$ is $(a-1 /(2(a+b)), \infty)$, if we choose

$$
x \leqslant a-\frac{1}{2(a+b)}
$$

then the inequality (55) always holds.

Therefore, when $a+b<0$, the series (53) is convergent if (56) holds.

For example, let $a=0$ and $b=1$, then $a+b>0$, from (54) we have $x \geqslant-1 / 2$, and (53) becomes the following convergent series with rational terms:

$$
\frac{1}{1+x}=1-\sum_{k=1}^{\infty} \frac{x^{k}}{(1+x)^{k+1}}, x \geqslant-1 / 2
$$

which is different from Taylor's series in the following:

$$
\frac{1}{1+x}=1+\sum_{k=1}^{\infty}(-1)^{n} x^{k},-1<x<1
$$

It is well known that the region of convergence of Taylor's series for the function $1 /(1+x)$ is $x \in(-1,1)$, which is much less than the region $x \in$ $[-1 / 2, \infty)$ obtained by series (57). Similar to the result of Example 6.1, when $x \notin([-1 / 2, \infty) \cap(-1,1))$ or $x \notin[-1 / 2,1)$, series $(57)$ and (58) are different functions.

On the other hand, the right-hand side of (57) still has the same pole as the function $1 /(1+x)$, but the right-hand side of (58) loses the important property.

\section{Conclusions}

The focus of this paper is on the general principle of the new form of expansion and infinite series for a given function, which is different from the Taylor polynomials and infinite series generated by the same given function.

As a new theory for expansion and infinite series, the work accomplished in this paper is favorable for the farther corresponding researches. Using the results proposed in this paper, we may obtain more achievements in many fields. Besides, the research methods and the results presented in this paper are 
academically valuable and have significances in many theories and applications, not only in mathematics but also in science and engineering.

It is well known that Taylors theorem has many applications in many fields. It implies that the new expansion and infinite series proposed in this paper can be extended to almost any fields to which the original form of Taylors expansion and infinite series can be applied. For example, we hope that this new theory can be extended to the fields of complex analysis, approximation, numeric analysis, engineering, physics, information science and so on, which wait for deeply investigating and exploring.

\section{References}

[1] G. Hardy, A Course of Pure Mathematics, Cambridge University Press Warehouse, London, 1908.

[2] T. M. Apostol, Mathematical Analysis, 2nd Edition, Pearson Education Asia Limited and China Machine Press, Beijing, 2004.

[3] H. S. Nik, F. Soleymani, A Taylor-type numerical method for solving nonlinear ordinary differential equations, Alexandria Engineering Journal, $\mathbf{5 2}$ (2013), 543-550.

[4] R. Barrio and M. Rodríguez and A. Abad and F. Blesa, Breaking the limits: The Taylor series method, Applied Mathematics and Computation, 217 (2011), 7940-7954.

[5] G. Groza, M. Razzaghi, A Taylor series method for the solution of the linear initial boundary-value problems for partial differential equations, Computers and Mathematics with Applications, 66 (2013), 1329-1343.

[6] K. Maleknejad, N. Aghazadeh, M. Rabbani, Numerical solution of second kind fredholm integral equations system by using a Taylor-series expansion method, Applied Mathematics and Computation, 175 (2006), 1229-1234.

[7] M. Masjed-Jamei, H. M. Srivastava, An integral expansion for analytic functions based upon the remainder values of the Taylor series expansions, em Applied Mathematics Letters, 22 (2009), 406-411.

[8] M. R. Eslahchi, M. Dehghan, Application of Taylor series in obtaining the orthogonal operational matrix, em Computers and Mathematics with Applications, 61 (2011), 2596-2604.

\section{Received: May 26, 2014}

